\title{
Determinants of variation in human serum paraoxonase activity
}

\author{
DL Rainwater ${ }^{1}$, S Rutherford ${ }^{1}$, TD Dyer ${ }^{1}$, ED Rainwater ${ }^{1}$, SA Cole ${ }^{1}$, JL VandeBerg ${ }^{1,2}$, L Almasy ${ }^{1}$, \\ J Blangero $^{1}$, JW MacCluer ${ }^{1}$ and MC Mahaney ${ }^{1,2}$ \\ ${ }^{1}$ Department of Genetics, Southwest Foundation for Biomedical Research, San Antonio, TX, USA and ${ }^{2}$ Southwest National Primate \\ Research Center, Southwest Foundation for Biomedical Research, San Antonio, TX, USA
}

\begin{abstract}
Paraoxonase-1 (PON1) is associated with high-density lipoprotein (HDL) particles and is believed to contribute to antiatherogenic properties of HDLs. We assessed the determinants of PON1 activity variation using different substrates of the enzyme. PON1 activity in serum samples from 922 participants in the San Antonio Family Heart Study was assayed using a reliable microplate format with three substrates: paraoxon, phenyl acetate and the lactone dihydrocoumarin. There were major differences among results from the three substrates in degree of effect by various environmental and genetic factors, suggesting that knowledge of one substrate activity alone may not provide a complete sense of PON1 metabolism. Three significant demographic covariates (age, smoking status and contra-
\end{abstract}

ceptive usage) together explained $1-6 \%$ of phenotypic variance, whereas four metabolic covariates representing lipoprotein metabolism (apoAll, apoAl, triglycerides and nonHDL cholesterol) explained 4-19\%. Genes explained 65$92 \%$ of phenotypic variance and the dominant genetic effect was exerted by a locus mapping at or near the protein structural locus (PON1) on chromosome 7. Additional genes influencing PON1 activity were localized to chromosomes 3 and 14. Our study identified environmental and genetic determinants of PON1 activity that accounted for $88-97 \%$ of total phenotypic variance, suggesting that few, if any, major biological determinants are unrepresented in the models. Heredity (2009) 102, 147-154; doi:10.1038/hdy.2008.110; published online 29 October 2008

Keywords: PON1; genetic; linkage analysis; Mexican Americans

\section{Introduction}

Paraoxonase-1 (PON1) is a liver-derived glycoprotein that is secreted into the circulation. Its gene (PON1) is a member of a larger gene family of PONs, which includes two other expressed proteins (PON2 and PON3), but PON1 is the dominant PON activity in blood (Draganov, 2007; Vaisar et al., 2007). PON1 is capable of hydrolyzing a diverse array of substrates, including a variety of esters, lactones and man-made organophosphate compounds. Enzymatic characterization has suggested that PON1 evolved as a lactonase (Draganov et al., 2005; Khersonsky and Tawfik, 2005). PON1 can also hydrolyze $\mathrm{N}$-acyl-homoserine lactones, which are quorum-sensing signals of pathogenic bacteria (Draganov et al., 2005).

Apart from a possible physiological role in protection from infectious diseases implied by the latter observation, PON1 has garnered much attention as a result of its ability to hydrolyze metabolites of toxic organophosphorus insecticides, such as parathion and diazinon, and chemical warfare nerve agents, such as soman and sarin. In addition, extensive research suggests that PON1 plays a key role in protection from cardiovascular disease (Durrington et al., 2001; Aviram and Rosenblat,

Correspondence: Dr DL Rainwater, Department of Genetics, Southwest Foundation for Biomedical Research, PO Box 760549, San Antonio, TX 78245-0549, USA.

E-mail:david@sfbrgenetics.org

Received 28 April 2008; revised 15 August 2008; accepted 23 September 2008; published online 29 October 2008
2005). Indeed, a recent study has detailed the relationships between PON1 (both genotype and enzyme activity), systemic oxidative stress and prevalence and incidence of cardiovascular disease (Bhattacharyya et al., 2008). PON1 is associated with high-density lipoprotein (HDL) in the circulation and, through its antioxidant and anti-inflammatory attributes, may contribute importantly to the antiatherogenic properties of HDL.

There is substantial interindividual variation in PON1 activity levels within a population and this suggests the possibility that individuals having lower PON1 levels may be at augmented risk from environmental exposures. Earlier studies have identified a number of factors, both genetic and environmental, that influence PON1 activity and corresponding susceptibility to environmental toxins (Deakin and James, 2004; Aviram and Rosenblat, 2005; Costa et al., 2005). In this study of a population of Mexican Americans, we have characterized the factors that influence PON1 enzymatic activity determined with three commonly used substrates: paraoxon, phenyl acetate and dihydrocoumarin.

\section{Materials and methods}

\section{Study participants and samples}

Study participants were drawn from the San Antonio Family Heart Study, which is a genetic study of cardiovascular disease risk factors for a population of Mexican Americans living in and around San Antonio, TX, USA. We identified the probands without regard to health 
status; they and all their first-, second- and third-degree relatives were invited to attend our clinic between 1992 and 1995 (MacCluer et al., 1999). PON1 activity was determined in samples from approximately 1330 individuals. In this study, we analyzed a subset of 922 participants $(69 \%)$ for whom we had complete data on covariates, genotypes and three substrate activities.

At the clinic visit, we obtained a fasting blood sample to measure various biochemical and metabolic variables, and we administered a survey to collect sociodemographic and behavioral characteristics (collectively indicated hereafter as demographic variables). Blood was allowed to clot and serum samples were prepared by low-speed centrifugation, aliquoted as individual singleuse aliquots protected from oxidation and desiccation (Cheng et al., 1986), and stored at $-80^{\circ} \mathrm{C}$ until they were thawed for the assay of PON activity. EDTA plasma samples were prepared and stored similarly and these aliquots were used for lipid and lipoprotein determinations. Clinical procedures were reviewed and approved by the Institutional Review Board of the University of Texas Health Science Center at San Antonio, and all subjects gave written informed consent.

\section{PON1 activity assays}

Serum PON1 activity was assessed spectrophotometrically using several substrates of the enzyme in a microplate format based on published protocols (Richter et al., 2004). PON1 activity (PON1-para) was determined using paraoxon (Aldrich Chemical Co., St Louis, MO, USA) as described previously (Rainwater et al., 2005). Briefly, serum was assayed in $0.1 \mathrm{M}$ Tris- $\mathrm{HCl}(\mathrm{pH} 8.5)$ containing $2 \mathrm{mM} \mathrm{CaCl}_{2}$ and $2 \mathrm{mM}$ substrate; appearance of $p$-nitrophenol was monitored at $405 \mathrm{~nm}$. PON1 arylesterase (PON1-aryl) activity was determined using phenyl acetate (Aldrich) as substrate (Gan et al., 1991). Serum was assayed in $0.1 \mathrm{M}$ Tris- $\mathrm{HCl}$ ( $\mathrm{pH}$ 8.5) containing $2 \mathrm{mM} \mathrm{CaCl}_{2}$ and $2 \mathrm{mM}$ substrate; appearance of phenol was monitored at $270 \mathrm{~nm}$. PON1 lactonase (PON1-lact) activity was assayed using dihydrocoumarin as described previously (Draganov et al., 2000). Serum was assayed in $0.05 \mathrm{M}$ Tris- $\mathrm{HCl}(\mathrm{pH} 8.0)$ containing $2 \mathrm{mM}$ $\mathrm{CaCl}_{2}$ and $2 \mathrm{mM}$ substrate; appearance of 3-(2-hydroxyphenyl)propionate was monitored at $270 \mathrm{~nm}$. For the hydrolysis of each of the substrates, there was an absolute requirement for calcium in the assay. All assays were conducted at $30^{\circ} \mathrm{C}$; kinetic rates were estimated during the linear phase and converted to moles using $\varepsilon=18050 \mathrm{~mol}^{-1} \mathrm{~L} \mathrm{~cm}^{-1}$ for PON1-para, $\varepsilon=3010 \mathrm{~mol}^{-1} \mathrm{~L} \mathrm{~cm}^{-1}$ for PON1-aryl and $\varepsilon=1295 \mathrm{~mol}^{-1} \mathrm{~L} \mathrm{~cm}^{-1}$ for PON1lact. Enzyme activities were expressed in units of

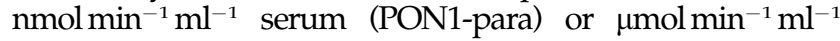
serum (PON1-aryl and PON1-lact). Each sample was run in duplicate wells and the average value was used in the analyses; the average coefficient of variation for duplicate samples was $1.1 \%$ for PON1-para, $1.1 \%$ for PON1-aryl and $2.2 \%$ for PON1-lact. Controls run on each plate gave betweenplate coefficients of variation of $6.3 \%$ for PON1-para, $7.9 \%$ for PON1-aryl and $6.6 \%$ for PON1-lact.

\section{Lipid and lipoprotein determinations}

Plasma cholesterol and triglyceride concentrations were determined by enzymatic assays performed with commercial reagents in a Ciba-Corning Express Plus clinical chemistry analyzer (Norwood, MA, USA) and apolipoprotein concentrations were determined by immunological assays as described previously (Rainwater et al., 2004). Lipoprotein size phenotypes were determined using nondenaturing gradient gel electrophoresis (Rainwater et al., 1997). To indicate global lipoprotein size distributions, we estimated HDL median diameter as the particle diameter where half of the HDL absorbance (occurring between 7.2 and $12.9 \mathrm{~nm}$ ) was on larger, and half was on smaller particles; similarly, low-density lipoprotein (LDL) median diameter was calculated for the LDL absorbance (occurring between 21 and $29 \mathrm{~nm}$ ).

\section{Genotypes}

DNA was prepared from lymphocytes and used in PCR with fluorescently labeled primers from the MapPairs Human Screening set, versions 6 and 8 (Research Genetics, Huntsville, AL, USA), which amplify 417 microsatellite markers spaced at approximately $10-\mathrm{cM}$ intervals across the 22 autosomes. Reactions were performed separately and products were pooled into multiplexed panels for typing on an automated DNA sequencer (ABI Model 377 with Genescan and Genotyper software; Applied Biosystems, Foster City, CA, USA). The average heterozygosity index for these markers was approximately 0.76. Because of the well-documented contributions of incorrect genotypes to type I and II errors, we employed a rigorous protocol to eliminate genotyping errors as described elsewhere (Rainwater et al., 2004). The sex-averaged marker map was based on that of deCODE genetics (Kong et al., 2002); those typed markers not on this map were placed by interpolation based on physical location (Göring et al., 2007). Multipoint identity-by-descent matrices were estimated using Markov chain Monte Carlo methods implemented in Loki (Heath, 1997).

Q192R (rs662) and L55M (rs854560) PON1 polymorphisms were typed using primers and probes from the Applied Biosystems Predesigned Drug Metabolism Genotyping Assays program (Applied Biosystems). Genotyping was performed with the ABI Prism 7900HT Sequence Detection System and typing errors were reduced using Simwalk2 (Sobel and Lange, 1996). The Q192R genotypes agreed well (97\% concordant) with our previous assessments based on PON1 activity status (Richter et al., 2004), and in a subset of unrelated individuals, both polymorphisms were in Hardy-Weinberg equilibrium.

\section{Statistical genetic analyses}

We employed the software SOLAR (Almasy and Blangero, 1998) to conduct all statistical genetic analyses; the traits comprised PON1 activity measured with three substrates. Initially, we used the covariate screen routine in SOLAR to test various demographic and lipid and lipoprotein phenotypes as potential covariates. This screen identified seven covariates significant for at least one of the three traits; thereafter, for any one type of model, the same set of covariates was applied for each trait regardless of significance level. We also included the indicator variables in some of the models to adjust for the PON1 polymorphisms Q192R and L55M, which result in nonsynonymous amino-acid substitutions. Genetic analyses employed a maximum likelihood-based variance 
decomposition approach implemented in SOLAR. We used this approach to partition the phenotypic variance in each trait $\left(\sigma_{\mathrm{P}}^{2}\right)$ into components representing additive genetic effects ( $\sigma_{\mathrm{G}}^{2}$, estimated as a function of relatedness) and environmental effects $\left(\sigma_{\mathrm{E}}^{2}\right)$. Residual heritability $\left(h^{2}\right)$ was estimated as the proportion of residual phenotypic variance (after covariate adjustment) that was attributable to additive genetic effects (that is, $h^{2}=\sigma_{\mathrm{G}}^{2} / \sigma_{\mathrm{P}}^{2}$ ). Significance was tested by comparing the $\log _{\mathrm{e}}$-likelihood ratio for the restricted model against that of the general model.

Genome-wide multipoint linkage analyses were performed as described previously (Almasy and Blangero, 1998). Briefly, we estimated the genetic variance attributable to the region around a given genetic marker $\left(\sigma_{\mathrm{Q}}^{2}\right)$ by specifying the expected genetic covariances between arbitrary relatives as a function of the identity-by-descent relationships at a marker locus assumed to be tightly linked to a gene influencing the quantitative trait. Genome-wide $P$-values were estimated using a modification of the method of Feingold et al. (1993) that takes into account the finite density of the marker loci in the map and the size and complexity of the pedigrees; for this population, logarithm of the odds (LOD) scores of $>2.9$ were judged to be significant evidence for a quantitative trait locus (QTL; Lander and Kruglyak, 1995). Oligogenic linkage analyses were performed in sequential fashion, with the QTL's location from the previous model fixed in the new model before the next genome-wide linkage scan (Blangero and Almasy, 1997). Before linkage analysis, traits were preadjusted for covariates and then fit to an inverse Gaussian distribution in SOLAR. This transformation yielded data that were both standardized and normalized, which better satisfies the assumptions of multivariate normality underlying variance decomposition approaches to linkage analysis. The estimates of proportion of the genetic variance associated with each QTL (and the residual) were taken from a linkage model in which all three QTL locations were fixed and the model maximized using SOLAR. However, the effect size estimates obtained at the locations of maximum statistical evidence for linkage can be grossly biased upward (Göring et al., 2001). This bias is inversely related to statistical power to localize the effect and, therefore, is likely reduced in this study compared with other studies (Blangero et al., 2003). Nevertheless, the bias still pertains to our estimates; consequently, we provide the estimates solely for their heuristic value.

\section{Results}

\section{Environmental covariate effects}

We included in this study samples from 922 participants for whom we had complete data for three PON1 substrate activities and associated covariates. We screened a number of potential covariates for effects on PON1 activity using the covariate screen routine in SOLAR. Demographic covariates that were tested included the effects of age, sex, smoking, diabetes, menopausal status and usage of various medications (for hyperlipidemia, blood pressure, diabetes, hormonal contraception and hormone replacement therapy). In addition, we screened a number of measures of lipoprotein metabolism for effects on PON1 activity. These metabolic covariates included concentrations of apoAI, apoAII, apoB, HDL cholesterol (HDLC), nonHDLC, and triglyceride as well as two indicators of lipoprotein size distributions, LDL and HDL median diameters. From these analyses, we identified seven covariates that exerted significant $(P<0.05)$ mean effects on at least one PON1 substrate. The significant demographic covariates were age, contraceptive usage, and smoking status and the metabolic covariates were concentrations of apoAI, apoAII, triglycerides, and nonHDLC. Table 1 shows, for each trait, mean, s.d. and $h^{2}$ estimated from analyses of the raw data; also shown are the standardized $\beta$-coefficients for each covariate, and the proportion of total phenotypic variance explained by covariates. Heritabilities for the three substrate activities were scaled to the covariate-adjusted residual variance and ranged from 78.4 to $97.9 \%$. The effects of three of the covariates-age, smoking status and apoAII concentrations-were consistently significant for all three traits, and of these, apoAII consistently exerted by far the strongest effects (Table 1). Contraceptive use, apoAI and non-HDLC were significant covariates for two of the three traits, but triglyceride concentrations were only significant for the PON1-aryl trait $(P=0.033)$.

Altogether, these seven environmental covariates explained differing proportions of the total phenotypic variance for each of the three substrates ranging from 5.2 to $25.1 \%$. We tested, with bivariate analyses, whether the proportion of phenotypic variance explained by covariates was significantly different for each of the three possible trait combinations. Accordingly, we compared the log-likelihoods for models in which the $\beta$-coefficients for each covariate were freely estimated for each trait to a model in which each coefficient was constrained to be the same across substrates. The raw data were normalized and standardized (by inverse Gaussian transformation), so we interpret significant differences (with 7

Table 1 Maximum likelihood estimates of mean $(\mu)$, s.d $(\sigma)$, heritability $\left(h^{2}\right)$, standardized $\beta$-coefficients and their s.e., associated $P$-values and proportion of total variance explained by covariates are given for PON1 activity levels for the three substrates

\begin{tabular}{|c|c|c|c|}
\hline Parameter & PON1-para & PON1-aryl & PON1-lact \\
\hline$\mu$ & $166.1 \pm 3.7$ & $82.3 \pm 1.1$ & $9.59 \pm 0.13$ \\
\hline$\sigma$ & $63.1 \pm 1.6$ & $18.6 \pm 0.5$ & $2.35 \pm 0.06$ \\
\hline$h^{2}, \%$ & $97.9 \pm 1.8$ & $85.2 \pm 5.6$ & $78.4 \pm 5.6$ \\
\hline$\beta$-Age & $-0.095 \pm 0.029^{*}$ & $-0.184 \pm 0.028^{* * *}$ & $-0.198 \pm 0.029^{* * *}$ \\
\hline$\beta$-Contraceptive & $0.029 \pm 0.026$ & $0.077 \pm 0.025^{*}$ & $0.080 \pm 0.025^{*}$ \\
\hline$\beta$-Smoking & $-0.089 \pm 0.027^{*}$ & $-0.102 \pm 0.026^{* *}$ & $-0.097 \pm 0.027^{* *}$ \\
\hline$\beta$-ApoAI & $0.046 \pm 0.036$ & $0.157 \pm 0.033^{* * *}$ & $0.155 \pm 0.034^{* * *}$ \\
\hline$\beta$-ApoAII & $0.244 \pm 0.036^{* * *}$ & $* 0.314 \pm 0.035^{* * *}$ & $0.236 \pm 0.036^{* * *}$ \\
\hline$\beta$-Triglycerides & $-0.001 \pm 0.042$ & $0.084 \pm 0.040^{*}$ & $0.002 \pm 0.041$ \\
\hline$\beta$-Non-HDLC & $0.064 \pm 0.036$ & $0.114 \pm 0.034^{* *}$ & $0.169 \pm 0.035^{* * *}$ \\
\hline $\begin{array}{l}\text { Covariate } \\
\text { effects, \% }\end{array}$ & 5.2 & 25.1 & 21.2 \\
\hline
\end{tabular}

Abbreviations: HDL, high-density lipoprotein; PON1, paraoxonase-1; PON1-aryl, PON1 arylesterase; PON1-lact, PON1 lactonase; PON1-para, paraoxonase activity of PON1.

PON1 activity units are nmol $\mathrm{min}^{-1} \mathrm{ml}^{-1}$ for PON1-para and $\mu \mathrm{mol} \mathrm{min}{ }^{-1} \mathrm{ml}^{-1}$ for PON1-aryl and PON1-lact. Standardized $\beta$-coefficients were estimated in separate models in which traits and covariates were standardized ( $Z$-score); key to $P$-values: ${ }^{*} P<0.05 ;{ }^{* *} P<0.001 ;{ }^{* * *} P<0.00001$. 
degrees of freedom) between the contrasting models to indicate significantly different proportions of variance explained by the common set of covariates. Covariate effects were significantly different for all pairs; $P$-values were $1.9 \times 10^{-16}, 2.6 \times 10^{-4}$ and $3.4 \times 10^{-3}$ for PON1-para / PON1-aryl, PON1-para/PON1-lact and PON1-aryl/ PON1-lact, respectively.

Effects of the Q192R and L55M polymorphisms in PON1 Two protein polymorphisms in PON1-Q192R and L55M-have been reported in earlier studies to exert significant effects on PON1 activity. We typed these two polymorphisms in all subjects and determined that allele frequencies for the 922 individuals in this study were 54 and 46\% for Q192 and R192, and 76 and 24\% for L55 and M55, respectively. Individually, both polymorphisms exert significant effects on all three substrate activities. Table 2 shows that, compared to R192/R192, Q192/Q192 homozygotes had lower activities for PON1-para and PON1-aryl, but higher levels for PON1-lact, whereas, compared to M55/M55, L55/L55 homozygotes had higher activities for all three substrates. The M55 variant is associated with lower PON1 activity levels probably as a result of strong disequilibrium with the T-108 and Q192 PON1 variants (Holland et al., 2006; Roest et al., 2007). Among the three substrate activities, the most pronounced genotype effects were for PON1-para.

\section{Linkage analyses}

We conducted genome-wide linkage screens using the three substrate activities that had been adjusted for the indicated covariates and then normalized to an inverseGaussian distribution. We tested three models for each substrate: one in which data had been adjusted only for the three demographic covariates (model 1), one in which data had been adjusted for these plus the four covariates expected to be related to PON1 metabolism (model 2) and one in which data had been adjusted for all covariates plus the Q192R and L55M polymorphisms (model 3). Table 3 presents the results of genome-wide multipoint linkage analyses for each of the nine models. The major linkage signal in each case was located on chromosome 7q21.11-21.3, presumably reflecting genetic variation at the structural locus for the enzyme, and the linkage peaks were flanked by markers D7S820 $(99.46 \mathrm{cM})$ and D7S821 $(107.94 \mathrm{cM})$ in each case. For each substrate, inclusion of the metabolic covariates strengthened, by more than 11 LOD units, the evidence for this QTL (comparing models 1 and 2). We recovered the major linkage signal on chromosome 7 even in model 3, which adjusted for two well-known PON1 protein polymorphisms, suggesting additional genetic variation at or near PON1 influences PON1 activity.

Next, we conducted a second genome-wide linkage screen conditional on the chromosome 7 QTL to detect any secondary genes. These analyses identified a second QTL (with weaker effects) influencing residual PON1 activity, but the location within the genome for the second QTL differed across the substrates. For example, a secondary, but significant, QTL for PON1-para was located on chromosome 3p22.3 (model 2), but there was no evidence for this QTL affecting either PON1-aryl or PON1-lact. Conversely, a significant secondary QTL for PON1-lact was located on chromosome 14q12-24.3 (model 2), but there was no significant support for this QTL with the other substrate activities. Flanking markers for these two signals were D3S3038 $(44.66 \mathrm{cM})$ and D3S2432 $(59.09 \mathrm{cM})$ for the former and D14S297 $(30.52 \mathrm{cM})$, and D14S588 $(69.65 \mathrm{cM})$ for the latter. Figure 1 shows a plot of multipoint LOD scores from model 2 for each of the substrate activities on chromosomes 3 (upper panel) and 14 (lower panel). Compared to that on

Table 3 Chromosomal (Chr) locations and LOD scores are indicated for primary and secondary multipoint LOD score peaks from analyses of covariate-adjusted and normalized PON1 activities for the three substrates

\begin{tabular}{|c|c|c|c|c|c|}
\hline \multirow[t]{2}{*}{ Substrate } & \multirow[t]{2}{*}{ Model } & \multicolumn{2}{|c|}{ Primary QTL } & \multicolumn{2}{|c|}{ Secondary QTL } \\
\hline & & $C h r(c M)$ & $L O D$ & Chr (cM) & $L O D$ \\
\hline PON1-para & 1 & 7q (105) & 23.0 & $3 p(70)$ & 2.9 \\
\hline PON1-para & 2 & 7q (104) & 38.1 & $3 p(59)$ & 3.4 \\
\hline PON1-para & 3 & 7q (106) & 10.7 & $14 q(43)$ & 2.2 \\
\hline PON1-aryl & 1 & 7q (104) & 8.9 & $1 \mathrm{p}(33)$ & 1.9 \\
\hline PON1-aryl & 2 & 7q (105) & 20.1 & $1 \mathrm{p}(32)$ & 2.8 \\
\hline PON1-aryl & 3 & 7q (106) & 15.7 & $1 \mathrm{p}(32)$ & 2.7 \\
\hline PON1-lact & 1 & 7q (105) & 16.5 & $14 q(32)$ & 1.9 \\
\hline PON1-lact & 2 & $7 q(105)$ & 28.3 & $14 q(48)$ & 3.4 \\
\hline PON1-lact & 3 & 7q (107) & 18.5 & $14 q(48)$ & 2.5 \\
\hline
\end{tabular}

Abbreviations: LOD, logarithm of the odds; PON1, paraoxonase-1; PON1-aryl, PON1 arylesterase; PON1-lact, PON1 lactonase; PON1para, paraoxonase activity of PON1.

Model 1, demographic covariates only; model 2, model 1 plus metabolic covariates; model 3, model 2 plus Q192R and L55M genotype information.

LOD scores in bold are significant at the genome-wide level.

Table 2 Mean \pm s.e.m. (and interquartile range) as a function of Q192R and L55M polymorphisms are given for raw PON1 activity levels for the three substrates

\begin{tabular}{lccrr}
\hline Genotype & $\mathrm{N}$ & PON1-para & PON1-aryl & PON1-lact \\
\hline Q192R & & & & \\
Q192/Q192 & 267 & $109.3 \pm 2.2(86-128)$ & $79.0 \pm 1.3(64-91)$ & $10.6 \pm 0.2(9-12)$ \\
Q192/R192 & 463 & $167.7 \pm 2.1(140-193)$ & $81.9 \pm 0.9(69-95)$ & $9.5 \pm 0.1(8-11)$ \\
R192/R192 & 192 & $229.6 \pm 4.5(194-267)$ & $85.7 \pm 1.6(73-101)$ & $8.4 \pm 0.2(7-10)$ \\
L55M & & & & \\
L55/L55 & 540 & $183.8 \pm 2.6(139-220)$ & $86.6 \pm 0.9(73-100)$ & \\
L55/M55 & 330 & $140.6 \pm 2.9(101-172)$ & $76.8 \pm 1.1(63-89)$ & $9.4 \pm 0.1(8-11)$ \\
M55/M55 & 52 & $100.7 \pm 7.0(74-117)$ & $65.2 \pm 2.3(57-76)$ & $8.4 \pm 0.3(7-10)$ \\
\hline
\end{tabular}

Abbreviation: PON1, paraoxonase-1; PON1-aryl, PON1 arylesterase; PON1-lact, PON1 lactonase; PON1-para, paraoxonase activity of PON1. 
Chromosome 3

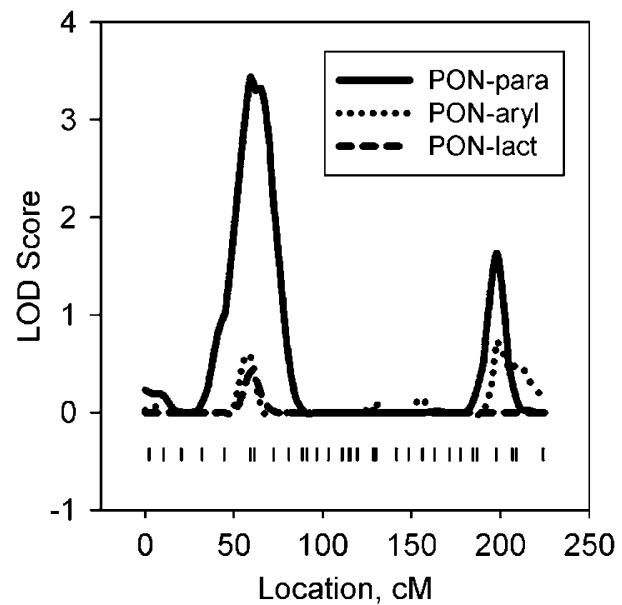

Chromosome 14

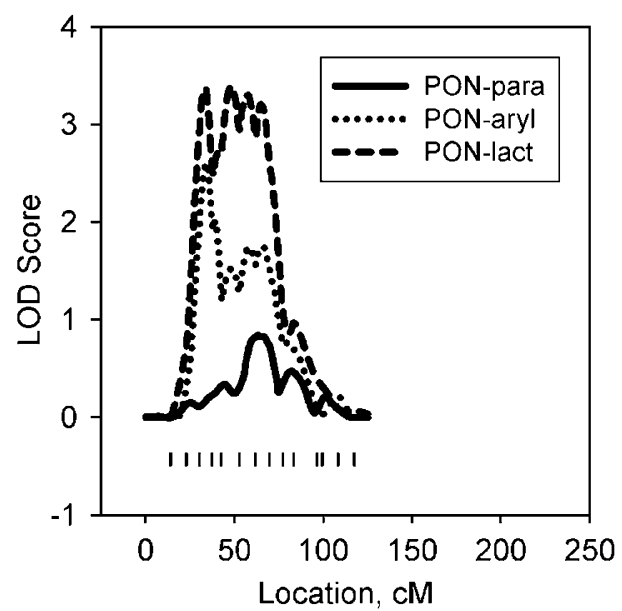

Figure 1 Multipoint logarithm of the odds (LOD) scores for three paraoxonase-1 (PON1) substrate activities plotted for chromosomes 3 and 14 . The tick marks indicate locations of microsatellite markers.

chromosome 3, the LOD score peak on chromosome 14 seems ill defined in the present map.

\section{Genetic effects on PON1 activity}

We conducted quantitative genetic analyses to approximate proportions of phenotypic variance associated with different determinants of PON1 activity. The models included the effects of the seven covariates on the normalized data and they also estimated variance associated with each of the QTLs (even if they were not significant). Table 4 presents the results of these analyses for each of the three substrate activities. As reported above, the covariate effects were relatively modest, accounting for $5-25 \%$ of total phenotypic variance, and differed significantly among the substrates. Genes accounted for $65 \%$ (PON1-aryl), 67\% (PON1-lact) and 92\% (PON1-para) of total phenotypic variance. By far, the largest component of genetic variance was explained by the QTL on chromosome 7, accounting for $60-78 \%$ of genetic variance (39-71\% of total phenotypic variance). The two secondary QTLs accounted for a much smaller proportion of the variance, estimated to range from $2-13 \%$ of phenotypic variance in the oligogenic models.
Table 4 Proportions (\%) of phenotypic variance for PON1-para, PON1-aryl and PON1-lact associated with covariates and genes

\begin{tabular}{lccc}
\hline & PON1-para & PON1-aryl & PON1-lact \\
\hline Environmental covariates & 5 & 25 & 21 \\
$\quad$ Demographic & 1 & 6 & 5 \\
Metabolic & \multicolumn{2}{c}{$\begin{array}{c}\text { PO } \\
\text { Genetic }\end{array}$} & \multicolumn{2}{c}{19} & 16 \\
QTL-chr7 & 92 & 65 & 67 \\
QTL-chr3 & 71 & 39 & 47 \\
QTL-chr14 & 8 & 4 & 2 \\
Residual & 4 & 12 & 13 \\
Unexplained & 9 & 10 & 5 \\
\hline
\end{tabular}

Abbreviations: chr, chromosome; PON1, paraoxonase-1; PON1-aryl, PON1 arylesterase; PON1-lact, PON1 lactonase; PON1-para, paraoxonase activity of PON1; QTL, quantitative trait locus.

Demographic covariates included age, contraceptive usage and smoking status; metabolic covariates included concentrations of apoAI, apoAII, triglycerides and non-HDLC. QTL effect sizes are subject to a strong upward bias, and therefore, the residual genetic component is likely to be underestimated.

In separate analyses, we estimated that the Q192R polymorphism accounted for approximately 48,1 and $8 \%$ of PON1-para, PON1-aryl and PON1-lact phenotypic variance, and that the L55M polymorphism accounted for 4,7 and $6 \%$, respectively (data not shown); these PON1 polymorphism effects would be included in the chromosome 7 QTL variance component in Table 4 . Residual genetic variation, not explained by the three QTLs, ranged from 5 to $10 \%$ of phenotypic variance, which likely is an underestimate of the true value given the upward bias in estimating the QTL effect sizes (Göring et al., 2001).

\section{Discussion}

In this study, we have attempted to identify the genetic and environmental determinants of variation in PON1 activity as measured with three substrates. Our final models, accounting for demographic, metabolic and genetic contributions, explained $88-97 \%$ of total phenotypic variation, depending on substrate. On the basis of these proportions, and considering the likelihood of some degree of assay error variance, we believe that there are few, if any, important biological determinants that are not represented in these models.

Demographic environmental covariates accounted for relatively little of the variation in PON1 activity, explaining 1-6\% of the total. Significant factors included contraceptive hormone usage (positive effect), age (negative effect) and smoking status (negative effect). We have previously reported significant positive effects of contraceptive usage on concentrations of apoAI, apoAII, triglycerides and cholesterol (Mitchell et al., 1996), suggesting that this effect on PON1 might be mediated through lipoprotein metabolism. Both smoking and age have been identified in other studies to have negative associations with PON1 (reviewed in (Deakin and James, 2004; Costa et al., 2005)). We were unable to test one recognized determinant of PON1 activity-diet. Studies in humans (Deakin and James, 2004; Aviram and Rosenblat, 2005), as well as our earlier studies in baboons (Rainwater et al., 2005), have demonstrated significant 
effects of various dietary components on PON1 activity. Although we were unable to test for the effects of diet in this study, it is likely that some dietary effects are indirectly reflected in the measures of lipoprotein metabolism that were included in this study. Nevertheless, it is possible that some of the unexplained PON1 variance could have resulted from differences in dietary intake among participants.

In contrast to the demographic covariates, the metabolic covariates exerted substantial effects on PON1 activity, ranging from 4 to $19 \%$ of the total phenotypic variance. In fact, the demographic covariates explained approximately only a third of the variance explained by the metabolic covariates. These metabolic covariates (apoAI, apoAII, triglycerides,and non-HDLC) appear to reflect the metabolism of both LDLs and HDLs, though by far, the strongest correlate of PON1 activity was concentration of the HDL-specific protein, apoAII (Table 1). Earlier studies have reported a number of HDL indicators, including apoAII (Saha et al., 1991; Blatter Garin et al., 1994), to be positively correlated with PON1 activity and concentration. In contrast to this trend in humans, however, are observations that PON1 content is reduced in HDLs of overexpressing apoAII-transgenic mice (Castellani et al., 1997; Ribas et al., 2004), which would imply a negative association. What can be concluded at this point is that, of the metabolic covariates tested, apoAII concentrations best represent an element(s) of lipoprotein metabolism that exerts important environmental influence on PON1 variation.

In a sense, it is surprising that another environmental determinant of PON1 variation is the nature of the enzyme substrate itself. That is, generally one would expect interindividual differences in activity rank order to be preserved for each substrate activity tested, but PON1 does not follow this expectation. For example, earlier studies (Davies et al., 1996) have identified striking substrate-specific effects of the Q192R protein polymorphism. Depending on the substrate tested in this study, we observed the effect size for the Q192R polymorphism to range from 1 to $48 \%$; the latter, for PON1-para, is similar to the previously reported value of $59 \%$ (Bhattacharyya et al., 2008). In addition to this wellestablished substrate-specific effect, we also have observed differential covariate effects, both in terms of which covariates are significant and their relative importance, which depend on the substrate employed. These findings suggest that knowledge of enzyme levels by a single method, such as enzyme concentrations or activity directed against a single substrate, may not provide a complete physiological picture of enzyme function in vivo.

Paraoxonase-1 genetics have been studied intensively for many years (Playfer et al., 1976; Geldmacher-v. Mallinckrodt et al., 1979). Most genetic studies have focused on the structural locus for the enzyme (PON1), and a number of protein and promoter polymorphisms in the structural gene have been reported to affect PON1 activity (Jarvik et al., 2003; Li et al., 2003). Not surprisingly, genes also were found to exert a very strong effect on PON1 activity in this study, accounting for $65-92 \%$ of total phenotypic variation. Such genetic effects on total enzymic activity could represent variants that influence enzyme concentration (expression) or specific activity or both. Primarily, the genetic control of PON1 activity was localized to a single site on chromosome 7 , in the vicinity of the PON1 structural locus, PON1. Two other members of the paraoxonase multigene family, PON2 and PON3, also map to this same region. The three genes lie within an approximately 137000-bp interval in the human genome (based on the March 2006 Human Genome Assembly, NCBI Build 36.1) and so are indistinguishable by linkage mapping. PON3 is also expressed in serum (Draganov et al., 2000; Reddy et al., 2001), although at much reduced levels compared to PON1 (Draganov, 2007; Vaisar et al., 2007). In addition, compared to PON1, PON3 has substantially lower specific activities for the substrates paraoxon and phenyl acetate, but virtually an identical specific activity for the substrate dihydrocoumarin (Draganov et al., 2005). Thus, although PON1 is the dominant paraoxonase enzyme in serum, it is possible that PON3 contributes a non-negligible portion of serum PON-lact activity.

Evidence for the chromosome 7 QTL was strengthened substantially by the inclusion of metabolic covariates, suggesting that the locus exerts a direct effect on the enzyme rather than an indirect effect mediated through lipoprotein metabolism. Furthermore, inclusion of two PON1 protein polymorphisms dampened, but did not eliminate, the chromosome 7 QTL (models 3, Table 3), suggesting that the additional genetic variation at this locus harboring the paraoxonase multigene family may also influence PON1 activity. Several PON1 promoter polymorphisms have been reported to affect PON1 activity and these-and potentially other-polymorphisms may well account for this residual QTL. Future work will focus on identifying these additional polymorphisms and quantifying their effects on PON1 activity.

In addition to the expected effects of genetic variation at (or near) PON1, we also have detected significant effects of QTLs elsewhere in the genome. In terms of proportion of total phenotypic variances, these other genes exert at most the modest effects on PON1 activity, but they are, in several cases, significant at the genomewide level. In various models, significant QTLs on chromosomes 3 and 14 were detected (Table 3). Although we do not know the specific genes that might be responsible for these QTLs, this observation does illustrate the value of quantifying PON1 activity with multiple substrates, as one QTL was detected only with the substrate dihydrocoumarin (PON1-lact), whereas the other was detected with the substrate paraoxon (PON1para) but not dihydrocoumarin. Of course, this sort of result could stem from the effects of various errors in the data preventing (or facilitating) the detection of marginally significant QTLs. Alternately, this result could reflect true interindividual variation in metabolic control of PON1 by these genes. An example of this might be a gene that, directly or indirectly, influences the partitioning of PON1 between HDL-associated and HDL-nonassociated states, as this characteristic significantly affects PON1 activity for some, but not all, substrates (Gaidukov and Tawfik, 2007).

In an earlier study of PON1-para alone, we detected an additional secondary QTL, located on chromosome 12p13.2-12.3 (Winnier et al., 2006), which was not observed in this study. We are not certain of the reason for this difference, but presume it to stem from differences in the precise set of individuals included, 
and the sets of covariates employed, in the two studies. However, the two studies do agree that a number of genes, other than the structural locus for PON1 on chromosome 7, exert important and significant effects on PON1 activity. Given the extensive adjustments for elements of lipoprotein metabolism in this study, it seems likely that the secondary genes on chromosomes 3 and 14 do not influence PON1 indirectly through the effects on lipoprotein metabolism.

There are several conclusions that may be drawn from this study in which we have accounted for $>88 \%$ of the total phenotypic variance. First, there are substantial environmental effects on PON1 variation, and a major environmental determinant is lipoprotein metabolism. Second, the proportional effects of various PON1 determinants differs by substrate, and this implies that knowledge of a single aspect of PON1 phenotype may not give a complete picture of the role PON1 may play in metabolism and susceptibility to organophosphate poisoning or cardiovascular disease. Finally, PON1 primarily is controlled by variation at the structural gene, but also by variation at several other unidentified genes.

\section{Acknowledgements}

The excellent technical assistance of Perry H Moore, Jr, Jane F VandeBerg, and Joel S Whitehead are gratefully acknowledged. This work was supported by a grant from NIH (P01 HL045522) and conducted in facilities constructed with support from the Research Facilities Improvement Program of NIH (C06 RR013556 and C06 RR017515); the SOLAR package is supported by R37 MH059490.

\section{References}

Almasy L, Blangero J (1998). Multipoint quantitative-trait linkage analysis in general pedigrees. Am J Hum Genet 62: 1198-1211.

Aviram M, Rosenblat M (2005). Paraoxonases and cardiovascular diseases: pharmacological and nutritional influences. Curr Opin Lipidol 16: 393-399.

Bhattacharyya T, Nicholls SJ, Topol EJ, Zhang R, Yang X, Schmitt D et al. (2008). Relationship of paraoxonase 1 (PON1) gene polymorphisms and functional activity with systemic oxidative stress and cardiovascular risk. JAMA 299: 1265-1276.

Blangero J, Almasy L (1997). Multipoint oligogenic linkage analysis of quantitative traits. Genet Epidemiol 14: 959-964.

Blangero J, Williams JT, Almasy L (2003). Novel family-based approaches to genetic risk in thrombosis. J Thromb Haemost 1: 1391-1397.

Blatter Garin MC, Abbott C, Messmer S, Mackness M, Durrington P, Pometta D et al. (1994). Quantification of human serum paraoxonase by enzyme-linked immunoassay: population differences in protein concentrations. Biochem J 304 (Part 2): 549-554.

Castellani LW, Navab M, Van Lenten BJ, Hedrick CC, Hama SY, Goto AM et al. (1997). Overexpression of apolipoprotein all in transgenic mice converts high density lipoproteins to proinflammatory particles. J Clin Invest 100: 464-474.

Cheng M-L, Woodford SC, Hilburn JL, VandeBerg JL (1986). A novel system for storage of sera frozen in small aliquots. J Biochem Biophys Methods 13: 47-51.

Costa LG, Vitalone A, Cole TB, Furlong CE (2005). Modulation of paraoxonase (PON1) activity. Biochem Pharmacol 69: 541-550.
Davies HG, Richter RJ, Keifer M, Broomfield CA, Sowalla J, Furlong CE (1996). The effect of the human serum paraoxonase polymorphism is reversed with diazoxon, soman and sarin. Nat Genet 14: 334-336.

Deakin SP, James RW (2004). Genetic and environmental factors modulating serum concentrations and activities of the antioxidant enzyme paraoxonase-1. Clin Sci (Lond) 107: 435-447.

Draganov DI (2007). Human PON3, effects beyond the HDL: clues from human PON3 transgenic mice. Circ Res 100: 1104-1105.

Draganov DI, Stetson PL, Watson CE, Billecke SS, La Du BN (2000). Rabbit serum paraoxonase 3 (PON3) is a high density lipoprotein-associated lactonase and protects low density lipoprotein against oxidation. J Biol Chem 275: 33435-33442.

Draganov DI, Teiber JF, Speelman A, Osawa Y, Sunahara R, La Du BN (2005). Human paraoxonases (PON1, PON2, and PON3) are lactonases with overlapping and distinct substrate specificities. J Lipid Res 46: 1239-1247.

Durrington PN, Mackness B, Mackness MI (2001). Paraoxonase and atherosclerosis. Arterioscler Thromb Vasc Biol 21: 473-480.

Feingold E, Brown PO, Siegmund D (1993). Gaussian models for genetic linkage analysis using complete high- resolution maps of identity by descent. Am J Hum Genet 53: 234-251.

Gaidukov L, Tawfik DS (2007). The development of human sera tests for HDL-bound serum paraoxonase PON1 and its lipolactonase activity. J Lipid Res 48: 1637-1646.

Gan KN, Smolen A, Eckerson HW, La Du BN (1991). Purification of human serum paraoxonase/arylesterase. Evidence for one esterase catalyzing both activities. Drug Metab Dispos 19: 100-106.

Geldmacher-v. Mallinckrodt M, Hommel G, Dumbach J (1979). On the genetics of the human serum paraoxonase (EC 3.1.1.2). Hum Genet 50: 313-326.

Göring HHH, Curran JE, Johnson MP, Dyer TD, Charlesworth J, Cole SA et al. (2007). Discovery of expression QTLs using large-scale transcriptional profiling in human lymphocytes. Nat Genet 39: 1208-1216.

Göring HHH, Terwilliger JD, Blangero J (2001). Large upward bias in estimation of locus-specific effects from genomewide scans. Am J Hum Genet 69: 1357-1369.

Heath SC (1997). Markov chain Monte Carlo methods for radiation hybrid mapping. J Comput Biol 4: 505-515.

Holland N, Furlong C, Bastaki M, Richter R, Bradman A, Huen $\mathrm{K}$ et al. (2006). Paraoxonase polymorphisms, haplotypes, and enzyme activity in Latino mothers and newborns. Environ Health Perspect 114: 985-991.

Jarvik GP, Jampsa R, Richter RJ, Carlson CS, Rieder MJ, Nickerson DA et al. (2003). Novel paraoxonase (PON1) nonsense and missense mutations predicted by functional genomic assay of PON1 status. Pharmacogenetics 13: 291-295.

Khersonsky O, Tawfik DS (2005). Structure-reactivity studies of serum paraoxonase PON1 suggest that its native activity is lactonase. Biochemistry 44: 6371-6382.

Kong A, Gudbjartsson DF, Sainz J, Jonsdottir GM, Gudjonsson SA, Richardsson B et al. (2002). A high-resolution recombination map of the human genome. Nat Genet 31: 241-247.

Lander E, Kruglyak L (1995). Genetic dissection of complex traits: guidelines for interpreting and reporting linkage results. Nature Genet 11: 241-247.

Li HL, Liu DP, Liang CC (2003). Paraoxonase gene polymorphisms, oxidative stress, and diseases. J Mol Med 81: 766-779.

MacCluer JW, Stern MP, Almasy L, Atwood LA, Blangero J, Comuzzie AG et al. (1999). Genetics of atherosclerosis risk factors in Mexican Americans. Nutr Rev 57: S59-S65.

Mitchell BD, Kammerer CM, Blangero J, Mahaney MC, Rainwater DL, Dyke B et al. (1996). Genetic and environmental contributions to cardiovascular risk factors in Mexican Americans. The San Antonio Family Heart Study. Circulation 94: 2159-2170. 
Playfer JR, Eze LC, Bullen MF, Evans DA (1976). Genetic polymorphism and interethnic variability of plasma paroxonase activity. J Med Genet 13: 337-342.

Rainwater DL, Mahaney MC, VandeBerg JL, Brush G, Almasy $\mathrm{L}$, Blangero J et al. (2004). A quantitative trait locus influences coordinated variation in measures of ApoB-containing lipoproteins. Atherosclerosis 176: 379-386.

Rainwater DL, Mahaney MC, Wang XL, Rogers J, Cox LA, VandeBerg JL (2005). Determinants of variation in serum paraoxonase enzyme activity in baboons. J Lipid Res 46: $1450-1456$.

Rainwater DL, Moore Jr PH, Shelledy WR, Dyer TD, Slifer SH (1997). Characterization of a composite gradient gel for the electrophoretic separation of lipoproteins. J Lipid Res 38: 1261-1266.

Reddy ST, Wadleigh DJ, Grijalva V, Ng C, Hama S, Gangopadhyay A et al. (2001). Human paraoxonase-3 is an HDL-associated enzyme with biological activity similar to paraoxonase-1 protein but is not regulated by oxidized lipids. Arterioscler Thromb Vasc Biol 21: 542-547.

Ribas V, Sanchez-Quesada JL, Anton R, Camacho M, Julve J, Escola-Gil JC et al. (2004). Human apolipoprotein A-II enrichment displaces paraoxonase from HDL and impairs its antioxidant properties: a new mechanism linking HDL protein composition and antiatherogenic potential. Circ Res 95: 789-797.

Richter RJ, Jampsa RL, Jarvik GP, Costa LG, Furlong CE (2004). Determination of paraoxonase 1 status and genotypes at specific polymorphic sites. Curr Protoc Toxicol 4: 4.12.1-4.12.19.

Roest M, van Himbergen TM, Barendrecht $\mathrm{AB}$, Peeters $\mathrm{PH}$, van der Schouw YT, Voorbij HA (2007). Genetic and environmental determinants of the PON-1 phenotype. Eur J Clin Invest 37: 187-196.

Saha N, Roy AC, Teo SH, Tay JS, Ratnam SS (1991). Influence of serum paraoxonase polymorphism on serum lipids and apolipoproteins. Clin Genet 40: 277-282.

Sobel E, Lange K (1996). Descent graphs in pedigree analysis: applications to haplotyping, location scores, and markersharing statistics. Am J Hum Genet 58: 1323-1337.

Vaisar T, Pennathur S, Green PS, Gharib SA, Hoofnagle AN, Cheung MC et al. (2007). Shotgun proteomics implicates protease inhibition and complement activation in the antiinflammatory properties of HDL. J Clin Invest 117: 746-756.

Winnier DA, Rainwater DL, Cole SA, Dyer TD, Blangero J, MacCluer JW et al. (2006). Multiple QTLs influence variation in paraoxonase 1 activity in Mexican Americans. Hum Biol 78: 341-352. 